SCORECARD

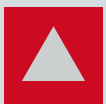

Fingerprint hackers

Hackers have published

what they claim is a

genuine copy of the fingerprint of German interior minister Wolfgang Schäuble, to protest against his plans to compile biometric data for the whole country.

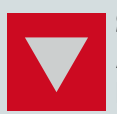

Spiteful hackers Another hacker group has been criticized for a less noble prank in which they hijacked the online forum of the Epilepsy Foundation, adding strobing graphics that triggered seizures in some users.

\section{NUMBER CRUNCH}

1979 is the year in which the World Health Organization declared smallpox officially extinct.

$\mathbf{8 7} \%$ of Scottish participants in a recent public survey conducted by the Society for General Microbiology in Reading, UK, had never heard of this medical triumph.

$40 \%$ of $16-24$-year-olds in the survey did not even know what microbes are.

\section{ON THE RECORD}

"I know a lot about sheep and cattle; I don't know much about satellites. But I would say it is a fuel cell off some stage of a rocket."

James Stirton, whose farm in Australia was hit by some space debris last year, clearly knows more about space aeronautics than he thinks.

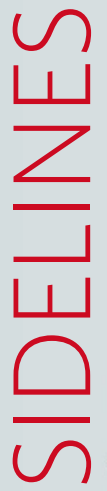

Sources: The Register, Wired, Society for General Microbiology,

Reuters

\title{
Easy ways to other Earths
}

A more precise way of calibrating the measurement of spectra should make it possible to identify Earth-sized planets around stars outside our Solar System using ground-based telescopes.

The technique, described on page 610 of this issue, makes use of laser 'combs'. Such combs are fine-toothed spectra of light that, when used in synch with atomic clocks, give scientists an exquisitely defined and stable reference point for measuring the wavelengths of light. The inventors of the laser comb shared half of the 2005 Nobel Prize in Physics, and the technology has made its mark in areas as diverse as chemical sensing and telecommunications. Now it can add astronomy to its list of applications.

'Astro-combs' should allow astronomers to measure the spectral lines of starlight with a precision as much as 60 times greater than the current state-of-the-art technique, say the authors of the paper, who are based at the Harvard-Smithsonian Center for Astrophysics in Cambridge, Massachusetts. As planets swing around stars, they induce movement that causes slight shifts in the stars' spectral lines. Astronomers have been using those shifts to infer the existence of planets for more than a decade, but until now have only been able to pick up the relatively large shifts caused by Jupiter-sized planets. The astro-combs should allow velocity measurements as precise

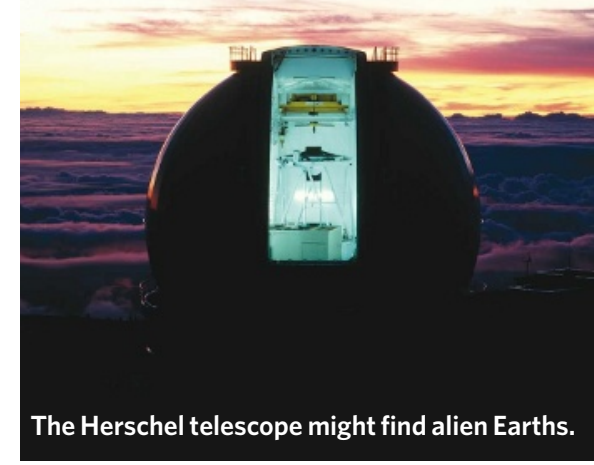

By June, the Harvard-Smithsonian group should have started testing its prototype system at the Multi-Mirror Telescope (MMT) Observatory on Mount Hopkins in Arizona. And, in 2009, the researchers plan to set up a planet-spotting system at the 4.2-metre William Herschel Telescope on La Palma, in the Canary Islands, in collaboration with the Geneva Observatory.

A rival group of researchers from the European Southern Observatory (ESO), which is based in Garching, Germany, says they have already used a similar astro-comb system to gather data with the Vacuum Tower Telescope on the neighbouring Canary Island of Tenerife. "Already we're showing that using these things on an astronomical telescope is a reality," says Michael Murphy, an astronomer at Swinburne University of Technology in Melbourne, Australia, and a collaborator with the ESO group.

The ESO team plans to use an astro-comb system at the observatory's 3.6-metre telescope at La Silla in Chile before eventually installing them at its four 8.2-metre telescopes at Cerro Paranal, also in Chile. "We came in as the fast, quick Americans who figured out a way to do just-good-enough calibration right away," says Walsworth of the Harvard team. "They're taking the MercedesBenz approach."

Whatever the approach, it will be as 1 centimetre per second, which brings the smaller shifts caused by Earth-sized planets into the detectible range.

Extending this technique's sensitivity will improve astronomers' picture of what other stellar systems look like. It will also provide targets for future work aimed at characterizing the atmospheres of any Earth-sized planets discovered, and at looking for indications of habitability.

Planet hunting is not the only task that the combs will make easier. If fitted with combbased systems, the coming generation of giant telescopes now on drawing boards might, over a period of decades, make direct measurements of the cosmic acceleration put down to 'dark energy?. "The impact of the technology will be huge," says Ronald Walsworth, a physicist at the Harvard-Smithsonian Center and a co-author of the astro-comb paper. a challenging technique to apply. For a start, planets are not the only things that move the surfaces of stars; they roil with starspots and pulse with starquakes. Such noise could render astro-combs moot, worries David Latham, a Harvard-Smithsonian astronomer. "It is a very large leap of faith that we can correct out the jitters in the stars themselves," he says. The combs' precision also makes strenuous demands on the stability of the spectrographs themselves. And because more photons are needed, more precise measurements will require either longer observation times or bigger telescopes.

Velocity measurements are not the only way to detect planets; they can also be seen by the changes they induce in the stars' position in the sky, and, in some cases, by the dimming they induce as they pass between their star and Earth. NASA's Kepler mission, due to launch in early 2009, aims to monitor the brightness of 
about 100,000 stars in a particular patch of sky in the hope of measuring such transits, something France's Corot satellite is already doing on a smaller scale. However, the stars Kepler will be looking at are almost all too distant for follow-up observations that might characterize its discoveries.

The other approach - making hyperaccurate measurements of a star's position - is the goal of NASA's US\$1.85-billion Space Interferometry Mission, or SIM, a bone of contention between the agency, which has sought to cut its funding, and its fans in the astronomical community. Zlatan Tsvetanov, the NASA programme scientist for SIM, says the astro-comb technique does not replace the SIM technique "by any means". But a forthcoming exoplanet report, commissioned by a National Science Foundation advisory committee, says that the number-one priority is finding another Earth in the most cost-effective way. If discovering Earth-sized planets with a technology that cost less than a million dollars really works, it may be hard for billion-dollar space missions to compete.

\section{Eric Hand}

See Q\&A, page xiii, and News \& Views, page 538.

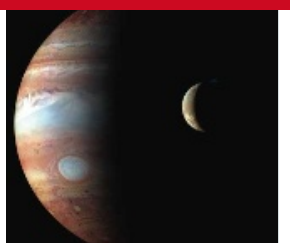

STORIES BY SUBJECT

Find all our space and physics news online www.nature.com/news/ archive/subject/index. html

\section{SNAPSHOT Flirty in pink}

Lustily trashing some hollowstemmed matting grass, this pink Amazonian river dolphin (Inia geoffrensis) is working it down the waterway in Mamirauá, a flooded rainforest reserve in Brazil. It's almost an exculsively male practice, this brandishing of grasses, sticks or clay. It is thought to be a courtship ritual, although no researcher has seen the dolphins mating, despite more than 13,000 hours of observation. If so, it places dolphins, with humans, chimps and birds, among the few animals that use objects as a flirting device.

Anna Petherick

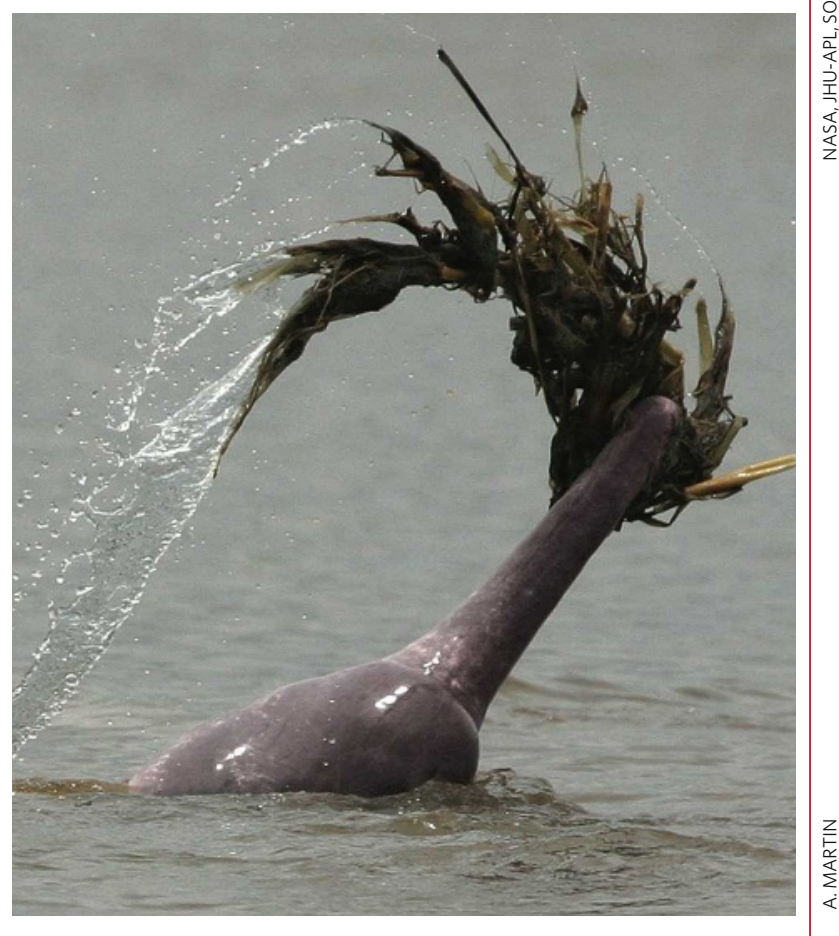

